\title{
THE GLOBAL GEOMETRY OF RIEMANNIAN MANIFOLDS WITH COMMUTING CURVATURE OPERATORS
}

\author{
M. BROZOS-VÁZQUEZ AND P. GILKEY
}

\begin{abstract}
We give manifolds whose Riemann curvature operators commute, i.e. which satisfy $\mathcal{R}\left(x_{1}, x_{2}\right) \mathcal{R}\left(x_{3}, x_{4}\right)=\mathcal{R}\left(x_{3}, x_{4}\right) \mathcal{R}\left(x_{1}, x_{2}\right)$ for all tangent vectors $x_{i}$ in both the Riemannian and the higher signature settings. These manifolds have global geometric phenomena which are quite different for higher signature manifolds than they are for Riemannian manifolds. Our focus is on global properties; questions of geodesic completeness and the behaviour of the exponential map are investigated.
\end{abstract}

\section{INTRODUCTION}

The study of curvature is central not only to Differential Geometry but to Global Analysis and Topology as well; one must relate properties of the curvature tensor to the underlying geometry and topology of the manifold under consideration the curvature tensor not only describes the geometry, but in a broad range of situations provides useful information about the topology and analytic properties of the manifold, especially when considering the global aspects. Much of this analysis involves examining a natural operator associated with the curvature; one studies commutativity or spectral properties of this operator. There are many operators which can be considered; the skew-symmetric curvature operator $\mathcal{R}(x, y)$ is perhaps the most natural one in this context. In this paper, we investigate a very natural geometric question by studying the global properties of manifolds which satisfy the condition

$$
\mathcal{R}\left(x_{1}, x_{2}\right) \mathcal{R}\left(x_{3}, x_{4}\right)=\mathcal{R}\left(x_{3}, x_{4}\right) \mathcal{R}\left(x_{1}, x_{2}\right)
$$

for all tangent vectors $x_{i}$-i.e. manifolds where the curvature operator is totally commutative.

The subject can properly have been said to have started with Osserman [14; seminal papers by Blažić et. al. [1, by Bonome et al. [2], by Chi 4], by Ivanov and Petrova [11, by Ivanova and Stanilov 12, by Nikolayevsky [13, by Stanilov [15]; by Stanilov and Videv [16, and by Tsankov [17] are only a few that could be mentioned; as the literature is a vast one, we must content ourselves for referring to the bibliographies in [6, 8, for further citations - the field is a vibrant one with a growing bibliography! A variety of methods, ranging from algebraic topology to elliptic operator theory and to classical invariance theory, have been used to study the spectral geometry of the curvature tensor.

Although the focus of this paper is on questions in global geometry, it is often convenient to study geometrical problems by first working in a purely algebraic context. One says that $\mathfrak{M}:=(V,\langle\cdot, \cdot\rangle, A)$ is a 0 -model if $V$ is a vector space of dimension $m$, if $\langle\cdot, \cdot\rangle$ is a non-degenerate symmetric bilinear form on $V$ of signature $(p, q)$, and if $A \in \otimes^{4} V^{*}$ is an algebraic curvature tensor on $V$; this means that $A$

1991 Mathematics Subject Classification. Primary 58B20; Secondary 53C20.

Key words and phrases. algebraic curvature tensor, Dunn manifold, exponential map, geodesic completeness, scalar curvature blow up, skew Tsankov manifold, warped product, Fiedler manifold. 
satisfies the usual curvature symmetries:

$$
\begin{aligned}
& A(x, y, z, w)=A(z, w, x, y)=-A(y, x, z, w), \\
& 0=A(x, y, z, w)+A(y, z, x, w)+A(z, x, y, w) .
\end{aligned}
$$

Let $\mathcal{A}$ be the associated skew-symmetric curvature operator:

$$
\langle\mathcal{A}(x, y) z, w\rangle=A(x, y, z, w) .
$$

One says that $A$ is skew Tsankov if

$$
\mathcal{A}(x, y) \mathcal{A}(u, v)=\mathcal{A}(u, v) \mathcal{A}(x, y) \quad \forall x, y, u, v .
$$

If $\mathcal{M}:=(M, g)$ is a pseudo-Riemannian manifold, let $R$ be the curvature tensor of the Levi-Civita connection and let the associated 0-model be given by:

$$
\mathfrak{M}(\mathcal{M}, P):=\left(T_{P} M, g_{P}, R_{P}\right) .
$$

One says that $\mathcal{M}$ is skew Tsankov if $\mathfrak{M}(\mathcal{M}, P)$ is skew Tsankov for every $P \in M$. The notation is motivated by the seminal result of Tsankov [17] who, following foundational suggestions of Stanilov, studied similar questions for hypersurfaces in $\mathbb{R}^{m}$; Tsankov imposed an extra condition of orthogonality that we shall not impose here. Similar questions arise for the Jacobi operator; see [3] for further details.

Here is a brief guide to this paper. In Section 2 we give a complete classification of Riemannian $(p=0)$ skew Tsankov algebraic curvature tensors. In Section 3 we present a family of irreducible 3-dimensional Riemannian skew Tsankov manifolds. In Section 4, we present a family of irreducible 4-dimensional Riemannian skew Tsankov manifolds. These examples indicate that despite the fact that the algebraic classification is complete, the geometric classification promises to be more difficult; questions of global geometry turn out to be deeper than the corresponding algebraic questions in this instance.

We then turn our attention to the pseudo-Riemannian setting. It turns out that many manifolds which appeared in other settings are also skew Tsankov. Dunn manifolds were introduced in [5]; they are a family of neutral signature pseudoRiemannian manifolds which are Osserman, Ivanov-Petrova, and Szabó. In Section 5] we show these manifolds are skew Tsankov with skew-symmetric curvature operators which are nilpotent of order 2. Certain Fiedler manifolds have been shown to be nilpotent Osserman of arbitrarily high order [7. In Section [6] we show that Fiedler manifolds are also skew Tsankov and that their skew-symmetric curvature operator is nilpotent of order 3. Certain Nikčević manifolds are skew Tsankov as well; the verification that these manifolds in 9] are skew Tsankov in dimension $m=6$ and signature $(4,2)$ is relatively straightforward and will be omitted in the interests of brevity. Thus there are many examples of skew Tsankov manifolds in the higher signature context; these examples indicate that even in the algebraic setting, the classification is likely to be far more complicated and this is a fruitful subject for further inquiry.

If $V=V_{1} \oplus V_{2}$ is a non-trivial orthogonal direct sum decomposition of $V$ which induces a decomposition $A=A_{1} \oplus A_{2}$, then $\mathfrak{M}=(V,\langle\cdot, \cdot\rangle, A)$ is said to be decomposable and we write $\mathfrak{M}=\mathfrak{M}_{1} \oplus \mathfrak{M}_{2}$ where $\mathfrak{M}_{i}:=\left(V_{i},\left.\langle\cdot, \cdot\rangle\right|_{V_{i}}, A_{i}\right) ; \mathfrak{M}$ is said to be indecomposable otherwise. A pseudo-Riemannian manifold $\mathcal{M}=(M, g)$ is said to be reducible at a point $P \in M$ if there is a neighborhood $\mathcal{O}$ of $P$ in $M$ and a Cartesian product $\mathcal{O}=\mathcal{O}_{1} \times \mathcal{O}_{2}$ which induces an orthonormal decomposition $g_{\mathcal{O}}=g_{\mathcal{O}_{1}} \oplus g_{\mathcal{O}_{2}} ; \mathcal{M}$ is locally irreducible at $P$ if $\mathcal{M}$ is not reducible at $P$.

The manifolds of Section [5] and [6] are all geodesically complete; by contrast, the scalar curvature of the manifolds described in Sections 3 and 4 blows up in finite time along certain geodesics and thus these manifolds are necessarily geodesically incomplete and can not be embedded isometrically in a geodesically complete 
manifold. It is not known if there are any irreducible skew Tsankov Riemannian manifolds of dimension $m \geq 3$ which are geodesically complete.

\section{The Classification of Riemannian skew Tsankov algebraic CURVATURE TENSORS}

Theorem 2.1. Let $\mathfrak{M}:=(V,\langle\cdot, \cdot\rangle, A)$ be a Riemannian 0 -model.

(1) $\mathfrak{M}$ is skew Tsankov if and only if there exists a orthogonal direct sum decomposition $V=V_{1} \oplus \ldots \oplus V_{k} \oplus W$ decomposing $A=A_{1} \oplus \ldots \oplus A_{k} \oplus 0$ where $\operatorname{dim}\left(V_{i}\right)=2$ for all $i$.

(2) $\mathfrak{M}$ is skew Tsankov and indecomposable if and only if $\operatorname{dim}(V)=2$ and $A \neq 0$.

Proof. Suppose given an orthogonal direct sum decomposition $V=V_{1} \oplus \ldots \oplus V_{k} \oplus W$ so $A=A_{1} \oplus \ldots \oplus A_{k} \oplus 0$ where $\operatorname{dim}\left(V_{i}\right)=2$ for $1 \leq i \leq k$. Let $\left\{e_{i}^{1}, e_{i}^{2}\right\}$ be an orthonormal basis for $V_{i}$. Given $x, y \in V$, there exist coefficients $\varepsilon_{i}(x, y) \in \mathbb{R}$ with

$$
\mathcal{A}(x, y) \xi=\left\{\begin{array}{rll}
-\varepsilon_{i}(x, y) e_{i}^{2} & \text { if } & \xi=e_{i}^{1}, \\
\varepsilon_{i}(x, y) e_{i}^{1} & \text { if } & \xi=e_{i}^{2}, \\
0 & \text { if } & \xi \perp \operatorname{Span}\left\{e_{i}^{1}, e_{i}^{2}\right\}
\end{array}\right.
$$

We may then show $\mathfrak{M}$ is skew Tsankov by computing

$$
\mathcal{A}(x, y) \mathcal{A}(\bar{x}, \bar{y}) \xi=\left\{\begin{array}{rll}
-\varepsilon_{i}(x, y) \varepsilon_{i}(\bar{x}, \bar{y}) e_{i}^{1} & \text { if } & \xi=e_{i}^{1}, \\
-\varepsilon_{i}(x, y) \varepsilon_{i}(\bar{x}, \bar{y}) e_{i}^{2} & \text { if } \quad \xi=e_{i}^{2}, \\
0 & \text { if } \quad \xi \perp \operatorname{Span}_{1 \leq i \leq k}\left\{e_{i}^{1}, e_{i}^{2}\right\} .
\end{array}\right.
$$

Conversely, suppose that $\mathfrak{M}$ is skew Tsankov. One may simultaneously skewdiagonalize the collection $\{A(x, y)\}_{x, y \in V}$ of commuting skew-adjoint linear operators to find an orthonormal set $\left\{e_{i}^{1}, e_{i}^{2}\right\}$ and to find functions $\varepsilon_{i}(x, y)$ where $1 \leq$ $i \leq k$ so that Equation (2.a) holds. Extend this to a full orthonormal basis $\mathcal{B}:=\left\{e_{1}^{1}, e_{1}^{2}, \ldots, e_{k}^{1}, e_{k}^{2}, f_{1}, \ldots, f_{l}\right\}$ for $V$. Then the only non-zero entries in the curvature tensor relative to this base are $A\left(\cdot, \cdot, e_{i}^{1}, e_{i}^{2}\right)$ modulo the usual $\mathbb{Z}_{2}$ symmetry. Interchanging the first 2 entries with the last 2 entries shows the only non-zero curvatures are

$$
A\left(e_{i}^{1}, e_{i}^{2}, e_{j}^{1}, e_{j}^{2}\right) .
$$

On the other hand, if $i \neq j$, we can use the Bianchi identity to express

$$
A\left(e_{i}^{1}, e_{i}^{2}, e_{j}^{1}, e_{j}^{2}\right)=-A\left(e_{i}^{1}, e_{j}^{1}, e_{j}^{2}, e_{i}^{2}\right)-A\left(e_{i}^{1}, e_{j}^{2}, e_{i}^{2}, e_{j}^{1}\right)=0
$$

and thus the only non-zero curvatures are $a_{i}:=A\left(e_{i}^{1}, e_{i}^{2}, e_{i}^{2}, e_{i}^{1}\right)$. Thus setting $V_{i}:=\operatorname{Span}\left\{e_{i}^{1}, e_{i}^{2}\right\}$ yields the desired decomposition $A=A_{1} \oplus \ldots \oplus A_{k} \oplus 0$. Assertions (1) and (2) now follow.

\section{3-Dimensional IRREDUCiBle SKEW TSANKOV MANIFOLDS}

We construct irreducible 3 -dimensional examples by taking a product of the interval $(0, \infty)$ with a Riemann surface:

Theorem 3.1. Let $\mathcal{N}:=\left(N, g_{N}\right)$ be a Riemann surface which does not have constant sectional curvature +1 . Give $\mathcal{M}:=\left((0, \infty) \times N, g_{M}\right)$ the warped product metric $g_{M}:=d t^{2}+t^{2} g_{N}$ for $t \in(0, \infty)$. Then $\mathcal{M}$ is an irreducible skew Tsankov manifold with scalar curvature $\tau_{\mathcal{M}}=t^{-2}\left\{\tau_{\mathcal{N}}-2\right\}$.

Proof. Choose isothermal coordinates to express $d s_{N}^{2}=e^{2 \alpha}\left(d x_{1}^{2}+d x_{2}^{2}\right)$, at least locally. Let $\partial_{1}:=\partial_{x_{1}}$, let $\partial_{2}:=\partial_{x_{2}}$, and let $\partial_{3}:=\partial_{t}$. Let $\alpha_{i}:=\partial_{i}(\alpha)$ and $\alpha_{i j}:=\partial_{i} \partial_{j}(\alpha)$. We have

$$
g_{M}\left(\partial_{1}, \partial_{1}\right)=g_{M}\left(\partial_{2}, \partial_{2}\right)=t^{2} e^{2 \alpha} \text { and } g_{M}\left(\partial_{3}, \partial_{3}\right)=1 .
$$


The non-zero Christoffel symbols of the first kind must have at least one repeated index different from 3 :

$$
\begin{array}{lll}
\Gamma_{111}=\alpha_{1} t^{2} e^{2 \alpha}, & \Gamma_{112}=-\alpha_{2} t^{2} e^{2 \alpha}, & \Gamma_{113}=-t e^{2 \alpha}, \\
\Gamma_{121}=\Gamma_{211}=\alpha_{2} t^{2} e^{2 \alpha}, & \Gamma_{131}=\Gamma_{311}=t e^{2 \alpha}, & \\
\Gamma_{221}=-\alpha_{1} t^{2} e^{2 \alpha}, & \Gamma_{222}=\alpha_{2} t^{2} e^{2 \alpha}, & \Gamma_{223}=-t e^{2 \alpha}, \\
\Gamma_{122}=\Gamma_{212}=\alpha_{1} t^{2} e^{2 \alpha}, & \Gamma_{322}=\Gamma_{232}=t e^{2 \alpha} . &
\end{array}
$$

Since the metric is diagonal, we can raise indices to see:

$$
\begin{aligned}
& \nabla_{\partial_{1}} \partial_{1}=\alpha_{1} \partial_{1}-\alpha_{2} \partial_{2}-t e^{2 \alpha} \partial_{3}, \\
& \nabla_{\partial_{1}} \partial_{2}=\nabla_{\partial_{2}} \partial_{1}=\alpha_{2} \partial_{1}+\alpha_{1} \partial_{2}, \\
& \nabla_{\partial_{1}} \partial_{3}=\nabla_{\partial_{3}} \partial_{1}=t^{-1} \partial_{1}, \\
& \nabla_{\partial_{2}} \partial_{2}=-\alpha_{1} \partial_{1}+\alpha_{2} \partial_{2}-t e^{2 \alpha} \partial_{3}, \\
& \nabla_{\partial_{2}} \partial_{3}=\nabla_{\partial_{3}} \partial_{2}=t^{-1} \partial_{2} .
\end{aligned}
$$

It is now an easy exercise to determine the curvature operator; we shall omit details in the interests of brevity. One has:

$$
\begin{aligned}
& \mathcal{R}_{\mathcal{M}}\left(\partial_{1}, \partial_{2}\right) \partial_{1}=\left(\alpha_{11}+\alpha_{22}+e^{2 \alpha}\right) \partial_{2}, \\
& \mathcal{R}_{\mathcal{M}}\left(\partial_{1}, \partial_{2}\right) \partial_{2}=-\left(\alpha_{11}+\alpha_{22}+e^{2 \alpha}\right) \partial_{1}, \\
& \mathcal{R}_{\mathcal{M}}\left(\partial_{1}, \partial_{3}\right) \partial_{3}=\mathcal{R}_{\mathcal{M}}\left(\partial_{2}, \partial_{3}\right) \partial_{3}=0 .
\end{aligned}
$$

As the only non-zero curvature is $R_{\mathcal{M}}\left(\partial_{1}, \partial_{2}, \partial_{2}, \partial_{1}\right)=-t^{2} e^{2 \alpha}\left(\alpha_{11}+\alpha_{22}+e^{2 \alpha}\right)$, Theorem 2.1 implies that $\mathcal{M}$ is skew Tsankov. This calculation also yields

$$
\tau_{\mathcal{M}}=t^{-2}\left\{-2 e^{-2 \alpha}\left(\alpha_{11}+\alpha_{22}\right)-2\right\} .
$$

An analogous computation on $\mathcal{N}$ yields:

$$
\begin{array}{lll}
\Gamma_{111}=\alpha_{1} t^{2} e^{2 \alpha}, & \Gamma_{112}=-\alpha_{2} t^{2} e^{2 \alpha}, & \Gamma_{121}=\Gamma_{211}=\alpha_{2} t^{2} e^{2 \alpha} \\
\Gamma_{221}=-\alpha_{1} t^{2} e^{2 \alpha}, & \Gamma_{222}=\alpha_{2} t^{2} e^{2 \alpha}, & \Gamma_{122}=\Gamma_{212}=\alpha_{1} t^{2} e^{2 \alpha}
\end{array}
$$

so the Christoffel symbols of the second kind and the curvature are given by:

$$
\begin{array}{ll}
\nabla_{\partial_{1}} \partial_{1}=\alpha_{1} \partial_{1}-\alpha_{2} \partial_{2}, & \nabla_{\partial_{2}} \partial_{2}=-\alpha_{1} \partial_{1}+\alpha_{2} \partial_{2} \\
\nabla_{\partial_{1}} \partial_{2}=\nabla_{\partial_{2}} \partial_{1}=\alpha_{2} \partial_{1}+\alpha_{1} \partial_{2}, & \mathcal{R}_{\mathcal{N}}\left(\partial_{1}, \partial_{2}\right) \partial_{1}=\left(\alpha_{11}+\alpha_{22}\right) \partial_{2}
\end{array}
$$

Theorem 3.1 now follows; $\mathcal{M}$ is indecomposable because Range $\{\mathcal{R}\}=\operatorname{Span}\left\{\partial_{1}, \partial_{2}\right\}$ and because $\tau_{\mathcal{M}}=t^{-2}\left(\tau_{\mathcal{N}}-2\right)$ exhibits non-trivial dependence on $t$.

\section{Remark 3.2.}

(1) Let $f\left(x_{1}, x_{2}\right)$ be an isometric embedding of a Riemann surface $N$ in $S^{3} \subset$ $\mathbb{R}^{4}$. Define an embedding of $(0, \infty) \times N$ in $\mathbb{R}^{4}$ by setting $F(t, x):=t f(x)$. Theorem 3.1 may then be used to see the resulting hypersurface in $\mathbb{R}^{4}$ is skew Tsankov; such hypersurfaces appear in Tsankov [17.

(2) Choose a point $x \in N$ where $\tau_{\mathcal{N}}(x) \neq 2$ and let $\gamma_{x}(t):=t \times x$. Then $\gamma_{x}$ is a unit speed geodesic and $\lim _{t \rightarrow 0}\left|\tau_{\mathcal{M}}\left(\gamma_{x}(t)\right)\right|=\infty$. Thus $\mathcal{M}$ exhibits scalar curvature blowup at finite time. This shows $\mathcal{M}$ is geodesically incomplete and can not be embedded isometrically in a geodesically complete manifold. It is not known whether or not there exist irreducible complete skew Tsankov 3-dimensional manifolds.

\section{4-Dimensional IRReducible Skew-Tsankov ManifoldS}

We take a warped product metric with a flat base and a flat fiber. Denote the usual coordinates on $\mathbb{R}^{4}$ by $\left(x_{1}, x_{2}, x_{3}, x_{4}\right)$. Let $\partial_{i}:=\partial_{x_{i}}$ and let

$$
\mathcal{O}:=\left\{\left(x_{1}, x_{2}, x_{3}, x_{4}\right) \in \mathbb{R}^{4}: x_{3}>0, x_{4}>0\right\} .
$$


Theorem 4.1. For $\beta>0$, let $\mathcal{M}_{\beta}:=\left(\mathcal{O}, g_{\beta}\right)$ where

$$
\begin{array}{ll}
g_{\beta}\left(\partial_{1}, \partial_{1}\right)=x_{3}^{2}, & g_{\beta}\left(\partial_{2}, \partial_{2}\right)=\left(x_{3}+\beta x_{4}\right)^{2}, \\
g_{\beta}\left(\partial_{3}, \partial_{3}\right)=1, & g_{\beta}\left(\partial_{4}, \partial_{4}\right)=1 .
\end{array}
$$

(1) $\mathcal{M}_{\beta}$ is an indecomposable skew Tsankov manifold.

(2) The scalar curvature $\tau_{\mathcal{M}_{\beta}}=-2 x_{3}^{-1}\left(x_{3}+\beta x_{4}\right)^{-1}$.

(3) $\mathcal{M}_{\beta_{1}}$ is not isometric to $\mathcal{M}_{\beta_{2}}$ for $\beta_{1} \neq \beta_{2}$.

Proof. The non-zero Christoffel symbols are given by:

$$
\begin{array}{ll}
\Gamma_{113}=-x_{3}, & \Gamma_{131}=\Gamma_{311}=x_{3}, \\
\Gamma_{223}=-\left(x_{3}+\beta x_{4}\right), & \Gamma_{232}=\Gamma_{322}=x_{3}+\beta x_{4}, \\
\Gamma_{224}=-\beta\left(x_{3}+\beta x_{4}\right), & \Gamma_{242}=\Gamma_{422}=\beta\left(x_{3}+\beta x_{4}\right) .
\end{array}
$$

Since the metric is diagonal, we may raise indices to compute:

$$
\begin{aligned}
& \nabla_{\partial_{1}} \partial_{1}=-x_{3} \partial_{3}, \\
& \nabla_{\partial_{1}} \partial_{3}=\nabla_{\partial_{3}} x_{1}=x_{3}^{-1} \partial_{1}, \\
& \nabla_{\partial_{2}} \partial_{2}=-\left(x_{3}+\beta x_{4}\right) \partial_{3}-\beta\left(x_{3}+\beta x_{4}\right) \partial_{4}, \\
& \nabla_{\partial_{2}} \partial_{3}=\nabla_{\partial_{3}} \partial_{2}=\left(x_{3}+\beta x_{4}\right)^{-1} \partial_{2}, \\
& \nabla_{\partial_{2}} \partial_{4}=\nabla_{\partial_{4}} \partial_{2}=\beta\left(x_{3}+\beta x_{4}\right)^{-1} \partial_{2} .
\end{aligned}
$$

The curvature operator can now be determined; as before, we shall omit the detailed computations in the interests of brevity:

$$
\begin{aligned}
& \mathcal{R}\left(\partial_{1}, \partial_{2}\right) \partial_{1}=x_{3}\left(x_{3}+\beta x_{4}\right)^{-1} \partial_{2}, \\
& \mathcal{R}\left(\partial_{1}, \partial_{2}\right) \partial_{2}=-x_{3}^{-1}\left(x_{3}+\beta x_{4}\right) \partial_{1} .
\end{aligned}
$$

The remaining curvatures vanish so the only non-zero curvature is

$$
\mathcal{R}\left(\partial_{1}, \partial_{2}, \partial_{2}, \partial_{1}\right)=-x_{3}\left(x_{3}+\beta x_{4}\right)
$$

and hence $\mathcal{M}$ is skew Tsankov by Theorem [2.1] This establishes Assertion (1); Assertion (2) follows from the computations performed above.

Let $\mathcal{E}:=\operatorname{Range}\{\mathcal{R}\}=\operatorname{Span}\left\{\partial_{1}, \partial_{2}\right\}$ and let $\mathcal{F}:=\mathcal{E}^{\perp}=\operatorname{Span}\left\{\partial_{3}, \partial_{4}\right\}$. These spaces are invariantly defined. We have

$$
\begin{aligned}
& \ln |\tau|=\ln (2)-\ln \left(x_{3}\right)-\ln \left(x_{3}+\beta x_{4}\right), \\
& \left.\nabla^{2}\{\ln |\tau|\}\right|_{\mathcal{F}}=\left(\begin{array}{cc}
x_{3}^{-2}+\left(x_{3}+\beta x_{4}\right)^{-2} & \beta\left(x_{3}+\beta x_{4}\right)^{-2}, \\
\beta\left(x_{3}+\beta x_{4}\right)^{-2} & \beta^{2}\left(x_{3}+\beta x_{4}\right)^{-2}
\end{array}\right), \\
& \operatorname{det}\left(\left.\nabla^{2}\{\ln |\tau|\}\right|_{\mathcal{F}}\right)=\beta^{2} x_{3}^{-2}\left(x_{3}+\beta x_{4}\right)^{-2}=\frac{1}{4} \beta \tau_{\mathcal{M}_{\beta}}^{2} .
\end{aligned}
$$

This shows that $\beta$ is an isometry invariant of $\mathcal{M}_{\beta}$. Furthermore since $\left.H\right|_{\mathcal{F}}$ has rank $2, \mathcal{M}$ is irreducible.

Remark 4.2. As in the example described in Section 3 the scalar curvature blows up at finite time along the geodesic $\gamma(t)=(1,1, t, 1)$; thus $\mathcal{M}_{\beta}$ can not be isometrically embedded as an open subset of a complete manifold; it is not known whether or not every irreducible complete skew Tsankov Riemannian manifold is necessarily 2-dimensional.

\section{DUNN MANIFOLDS}

We study the following family of Dunn manifolds which was first introduced in [5] in a different context.

Theorem 5.1. Let $\left(x_{1}, \ldots, x_{p}, y_{1}, \ldots, y_{p}\right)$ be coordinates on $\mathbb{R}^{2 p}$. Let $\psi_{i j}(x)=\psi_{j i}(x)$ be given. Let $\mathcal{M}:=\left(\mathbb{R}^{2 p}, g\right)$ be the manifold of neutral signature $(p, p)$ where

$$
g\left(\partial_{x_{i}}, \partial_{x_{j}}\right)=\psi_{i j}(x) \quad \text { and } g\left(\partial_{x_{i}}, \partial_{y_{i}}\right)=1 \text {. }
$$

Then $\mathcal{M}$ is skew Tsankov and $\mathcal{R}$ is nilpotent of order 2. 
Proof. The non-zero Christoffel symbols are:

$$
\begin{aligned}
& g\left(\nabla_{\partial_{x_{i}}} \partial_{x_{j}}, \partial_{x_{k}}\right)=\frac{1}{2}\left(\psi_{i k / j}+\psi_{j k / i}-\psi_{i j / k}\right), \\
& \nabla_{\partial_{x_{i}}} \partial_{x_{j}}=\frac{1}{2} \sum_{k}\left(\psi_{i k / j}+\psi_{j k / i}-\psi_{i j / k}\right) \partial_{y_{k}} .
\end{aligned}
$$

From this it follows that the possibly non-zero entries in curvature tensor $R$ are:

$$
R_{i j k l}=-\frac{1}{2} \sum_{l}\left(\psi_{i l / j k}+\psi_{j k / i l}-\psi_{i k / j l}-\psi_{j l / i k}\right) .
$$

Consequently $\mathcal{R}\left(\partial_{x_{i}}, \partial_{x_{j}}\right) \partial_{x_{k}}=\sum_{\ell} R_{i j k l} \partial_{y_{\ell}}$. This shows that

$$
\text { Range }(\mathcal{R}) \subset \operatorname{Span}\left\{\partial_{y_{i}}\right\} \text { and } \operatorname{Span}\left\{\partial_{y_{i}}\right\} \subset \operatorname{Ker}(\mathcal{R}) \text {. }
$$

Thus $\mathcal{R}\left(\xi_{1}, \xi_{2}\right) \mathcal{R}\left(\xi_{3}, \xi_{4}\right)=0$ for all $\xi_{1}, \xi_{2}, \xi_{3}, \xi_{4}$ so $\mathcal{M}$ is skew Tsankov.

Remark 5.2. These manifolds have been studied extensively. These manifolds are all geodesically complete and the exponential map is a global diffeomorphism. If $\psi_{i j}=\partial_{x_{i}} f \partial_{x_{j}} f$ for some function $f$, then $\mathcal{M}$ is realizable as a hypersurface in $\mathbb{R}^{(p, p+1)}$. Certain manifolds in this family are curvature homogeneous but not homogeneous. We refer to [5] for further details. Thus, in contrast to the Riemannian setting, there are examples which are global in the sense that they are geodesically complete.

\section{Fiedler MANifolds}

The following family of examples was first introduced in [7].

Theorem 6.1. Let $\left(x, u_{1}, \ldots, u_{\nu}, y\right)$ be coordinates on $\mathbb{R}^{\nu+2}$. Let $f \in C^{\infty}\left(\mathbb{R}^{\nu}\right)$ and let $\Xi=\Xi_{a b}$ be an invertible symmetric $\nu \times \nu$ matrix of signature $(r, s)$. Define a metric $g$ of signature $(r+1, s+1)$ on $\mathbb{R}^{\nu+2}$ by setting:

$$
g\left(\partial_{x}, \partial_{x}\right)=-2 f(\vec{u}), \quad g\left(\partial_{x}, \partial_{y}\right)=1, \quad \text { and } \quad g\left(\partial_{u_{a}}, \partial_{u_{b}}\right)=\Xi_{a b} .
$$

Then $\mathcal{M}$ is skew Tsankov and $\mathcal{R}$ is nilpotent of order 3 .

Proof. Since $d \Xi=0$, the potentially non-zero Christoffel symbols are:

$$
\begin{aligned}
& g\left(\nabla_{\partial_{x}} \partial_{x}, \partial_{u_{a}}\right)=\partial_{u_{a}}(f), \\
& g\left(\nabla_{\partial_{u_{a}}} \partial_{x}, \partial_{x}\right)=g\left(\nabla_{\partial_{x}} \partial_{u_{a}}, \partial_{x}\right)=-\partial_{u_{a}}(f) .
\end{aligned}
$$

Let $\Xi^{a b}$ be the inverse matrix. Then

$$
\begin{aligned}
& \nabla_{\partial_{x}} \partial_{x}=\sum_{a b} \Xi^{a b} \partial_{u_{a}}(f) \partial_{u_{b}}, \\
& \nabla_{\partial_{x}} \partial_{u_{a}}=\nabla_{\partial_{u_{a}}} \partial_{x}=-\partial_{u_{a}}(f) \partial_{y} .
\end{aligned}
$$

The quadratic terms in the Christoffel symbols play no role in the calculation of $R$. Let $f_{a b}:=\partial_{u_{a}} \partial_{u_{b}} f$. The possibly non-zero components of $R$ and of $\mathcal{R}$ are

$$
\begin{aligned}
& R\left(\partial_{x}, \partial_{u_{a}}, \partial_{u_{b}}, \partial_{x}\right)=f_{a b}, \\
& \mathcal{R}\left(\partial_{x}, \partial_{u_{a}}\right) \partial_{u_{b}}=f_{a b} \partial_{y} \text { and } \mathcal{R}\left(\partial_{x}, \partial_{u_{a}}\right) \partial_{x}=-\Xi^{b c} f_{a c} \partial_{u_{b}} .
\end{aligned}
$$

Thus the only potentially non-zero quadratic terms in the curvature are

$$
\mathcal{R}\left(\partial_{x}, \partial_{u_{d}}\right) \mathcal{R}\left(\partial_{x}, \partial_{u_{a}}\right) \partial_{x}=-\Xi^{b c} f_{a c} f_{d b} \partial_{y} .
$$

It now follows that $\mathcal{R}\left(\partial_{x}, \partial_{u_{d}}\right) \mathcal{R}\left(\partial_{x}, \partial_{u_{a}}\right) \partial_{x}=\mathcal{R}\left(\partial_{x}, \partial_{u_{a}}\right) \mathcal{R}\left(\partial_{x}, \partial_{u_{d}}\right) \partial_{x}$. This shows that $\mathcal{M}$ is skew-Tsankov and that $\mathcal{R}$ is nilpotent of order 3 .

These manifolds are irreducible for generic $f$. They are complete for certain choices of the warping function but are not in general geodesically complete. Of particular interest is the Lorentzian case. We refer to [10] for the following results that again relate to the global geometry of these examples. Let $\mathcal{M}_{f}:=\left(\mathbb{R}^{3}, g_{f}\right)$ where $g_{f}$ is the Lorentz metric on $\mathbb{R}^{3}$ given by:

$$
g_{f}\left(\partial_{x}, \partial_{x}\right)=-2 f(y) \quad \text { and } \quad g_{f}\left(\partial_{x}, \partial_{\tilde{x}}\right)=g_{f}\left(\partial_{y}, \partial_{y}\right)=1 .
$$


Example 6.2. Let $\mathcal{S}_{\varepsilon}$ be defined by $f_{\varepsilon}(y):=\frac{1}{2} \varepsilon y^{2}$ for $\varepsilon= \pm 1$.

(1) The manifolds $\mathcal{S}_{ \pm}$are geodesically complete.

(2) The map $\exp _{P}$ for $\mathcal{S}_{+}$is not surjective $\forall P \in \mathbb{R}^{3}$.

(3) The map $\exp _{P}$ for $\mathcal{S}_{-}$is a global diffeomorphism from $T_{P} \mathbb{R}^{3}$ to $\mathbb{R}^{3} \forall P \in \mathbb{R}^{3}$.

We say that a pseudo-Riemannian manifold $\mathcal{M}$ is $k$-curvature homogeneous if given any two points $P$ and $Q$ of $M$, there is an isometry $\phi: T_{P} M \rightarrow T_{Q} M$ so that $\phi^{*} \nabla^{i} R_{Q}=\nabla^{i} R_{P}$ for $i \leq k$. We say $\mathcal{M}$ Ricci explodes if there exists a geodesic $\gamma$ defined for $t \in(0, T)$ so $\lim _{t \rightarrow 0}|\rho(\dot{\gamma}(t), \dot{\gamma}(t))|=\infty$.

Example 6.3. For $1 \leq i \leq 6$, let $\mathcal{N}_{i, \pm}:=\mathcal{M}_{f_{i, \pm}}$ where

$$
\begin{array}{lll}
f_{1,-}(y)=-e^{-y}, & f_{2,-}(y)=-e^{-y}+y, & f_{3,-}(y)=-e^{-y}-e^{-2 y}, \\
f_{1,+}(y)=e^{y}, & f_{2,+}(y)=e^{y}+y, & f_{3,+}(y)=e^{y}+e^{2 y} .
\end{array}
$$

(1) $\mathcal{N}_{1,-}$ is locally homogeneous and Ricci explodes.

(2) $\mathcal{N}_{2,-}$ is 1-curvature homogeneous, not 2-curvature homogeneous, and Ricci explodes.

(3) $\mathcal{N}_{3,-}$ is 0 -curvature homogeneous, not 1-curvature homogeneous, and Ricci explodes.

(4) $\mathcal{N}_{1,+}$ is geodesically complete, and homogeneous.

(5) $\mathcal{N}_{2,+}$ is 1-curvature homogeneous, not 2-curvature homogeneous, and geodesically complete.

(6) $\mathcal{N}_{3,+}$ is 0-curvature homogeneous, not 1-curvature homogeneous, and geodesically complete.

\section{ACKNOWLEDGMENTS}

The research of M. Brozos-Vázquez was partially supported by project BFM 2003-02949 (Spain). The research of both M. Brozos-Vázquez and P. Gilkey was partially supported by the Max Planck Institute for Mathematics in the Sciences (Leipzig, Germany).

\section{REFERENCES}

[1] N. Blažić, N. Bokan and Z. Rakić, A note on the Osserman conjecture and isotropic covariant derivative of curvature, Proc. Amer. Math. Soc. 128 (2000), 245-253.

[2] A. Bonome, P. Castro and E. García-Río, Four-Dimensional Generalized Osserman Manifolds, Classical Quantum Gravity 18 (2001), 4813-4822.

[3] M. Brozos-Vázquez and P. Gilkey, Manifolds with Commuting Jacobi Operators, to appear J. Geometry.

[4] Q. S. Chi, Curvature characterization of certain locally rank one symmetric spaces, J. Differential Geom. 28 (1988), 187-202.

[5] C. Dunn and P. Gilkey, Curvature homogeneous pseudo-Riemannian manifolds which are not locally homogeneous, Complex, contact and symmetric manifolds, Progr. Math. 234 Birkhäuser Boston, Boston, MA, (2005), 145-152.

[6] E. García-Río, D. Kupeli, and R. Vázquez-Lorenzo, Osserman Manifolds in SemiRiemannian Geometry, Lecture Notes in Mathematics, 1777, Springer-Verlag, Berlin, 2002.

[7] B. Fiedler and P. Gilkey, Nilpotent Szabó, Osserman and Ivanov-Petrova pseudoRiemannian manifolds, Contemporary Mathematics 337 (2003), 53-64.

[8] P. Gilkey, Geometric Properties of Natural Operators Defined by the Riemann Curvature Tensor, World Scientific (2001).

[9] P. Gilkey and S. Nikčević, Curvature homogeneous spacelike Jordan Osserman pseudoRiemannian manifolds, Class. Quantum Grav 21 (2004), 497-507.

[10] P. Gilkey and S. Nikčević, Affine curvature homogeneous 3-dimensional Lorentz Manifolds, International Journal of Geometric Methods in Modern Physics 2 (2005), 737-749.

[11] S. Ivanov and I. Petrova, Riemannian manifolds in which the skew-symmetric curvature operator has pointwise constant eigenvalues, Geom. Dedicata 70 (1998), 269-282.

[12] R. Ivanova and G. Stanilov, A skew-symmetric curvature operator in Riemannian geometry, Symposia Gaussiana (1994), Conf. A: Mathematics, Eds. Behara, Fritsch and Lintz (1995), 391-395. 
[13] Y. Nikolayevsky, Riemannian manifolds whose curvature operator $R(X, Y)$ has constant eigenvalues, Bull. Austral. Math. Soc. 70 (2004), 301-319.

[14] R. Osserman, Curvature in the eighties, Amer. Math. Monthly 97 (1990), 731-756.

[15] G. Stanilov, Higher order skew-symmetric and symmetric curvature operators, C. R. Acad. Bulgare Sci. 57 (2004), 9-12.

[16] G. Stanilov, and V. Videv, On a generalization of the Jacobi operator in the Riemannian geometry, Annuaire Univ. Sofia Fac. Math. Inform. 86 (1992), 27-34.

[17] Y. Tsankov, A characterization of $n$-dimensional hypersurfaces in $\mathbb{R}^{n+1}$ with commuting curvature operators, Banach Center Publ. 69 (2005), 205-209.

Department of Geometry and Topology, Faculty of Mathematics, University of Santiago de Compostela, 15782 Santiago de Compostela, Spain

E-mail address: mbrozos@usc.es

Mathematics Department, University of Oregon, Eugene, OR 97403, USA

E-mail address: gilkey@.uoregon.edu 